\title{
A Kinetic Energy Harvester with Fast Start-up for Wearable Body-Monitoring Sensors
}

\author{
Jacopo Olivo*, Davide Brunelli ${ }^{\dagger}$ and Luca Benini ${ }^{\ddagger}$ \\ *Integrated Systems Laboratory \\ Federal Technology Institute of Lausanne, Lausanne, Switzerland \\ email: jacopo.olivo@epfl.ch \\ ${ }^{\dagger}$ Department of Information Engineering and Computer Science \\ University of Trento, Trento, Italy \\ email: davide.brunelli@disi.unitn.it \\ ${ }^{\ddagger}$ Department of Electronics, Computer Sciences and Systems \\ University of Bologna, Bologna, Italy \\ email: luca.benini@unibo.it
}

\begin{abstract}
A kinetic energy harvester for wearable wireless sensors and personal devices is proposed. The harvester is based on electromagnetic transduction of human movements and on a charge pump with smart switching to minimize the startup time from the fully depleted state. A detailed analysis and design optimization in the frequency range typical of human motion is also presented. Experimental results confirm that the proposed approach sharply reduces the start-up time and can exploit efficiently even small movements.
\end{abstract}

\section{INTRODUCTION}

Research field of Body Area Networks (BAN) and their applications to healthcare monitoring are attracting increasing attention. The possibility to carefully monitor the human body, its movements, its activities and its health status paves the way to a wide variety of applications and offers interesting scenarios for the future. Smart environments, sensitive and responsive to the presence of people, represent an example of a possible application field. Wearable wireless sensors make it possible to collect information about individual and collective user behavior, and ultimately to anticipate their requests before they are expressed. Another strategic application domain is Ambient-Assisted Living (AAL): by collecting and sending in real time physiological data, it is possible to better calibrate a therapy or to prevent dangerous events, such as falls or heart attacks.

Several key challenges must be addressed to realize these scenarios. Body area networks for healthcare monitoring must be unobtrusive, easily wearable and must feature large autonomy in terms of battery life. Energy constraints, in particular, can be tackled by wearable energy harvesters, also called energy scavengers, transforming human movements into electric energy to recharge, or even substitute, the batteries.

The energy scavenger proposed in this paper collects the energy returned by a miniaturized electro-magnetic motor located on the human body and used as a generator. We have focused on a system which can power up a personal device or a wearable sensor faster than using a single supercapacitor as power supply. Although the market can provide supercapacitors with considerable capacitance values, able to store a large amount of energy, their use as power supplies is limited by the time they need to reach a useful voltage level when they are charged by low currents. To overcome this drawback, in our approach the charge obtained is initially stored into a supercapacitor and then transferred efficiently to a smaller accumulator to reduce the start-up time and to power immediately a sensor node.

The remainder of the paper is organized as follows: related works are reviewed in the next section, followed by a list of the contributions and innovations that our work proposes. Section 3 reports the description of the different parts of our system, including their sizing and functioning. Section 4 describes the measurements realized to validate the system: the performance of the energy scavenger has been compared against that of a single supercapacitor used as a simple battery, allowing us to estimate the benefits of our system. Finally, Section 5 concludes the paper.

\section{RELATED WORK}

Wearable energy scavengers for body area networks have been studied extensively and the literature describes many solutions for a large variety of applications. Most physical phenomena have been explored to obtain energy scavengers suitable for on-body applications, non-invasive and with high efficiency. Thermoelectric effect has been used to convert the heat generated by humans into electric energy, returning power in the order of few $\mu \mathrm{W} / \mathrm{cm}^{2}$ in the presence of thermal gradients below $5{ }^{\circ} \mathrm{C}[1,2]$. Infrared (IR) and radio-frequency (RF) radiations [3-5], as well as low frequency magnetic fields [6], can be exploited to power implanted biosensors, being able to deliver power wirelessly through the biological tissues. Fuel cells are another solution suitable for implanted biosensors [7]: by using chemical species already present into the body fluids, such as the glucose, these devices can generate power through electrochemical reactions without any external support. Most of these solutions need power management 


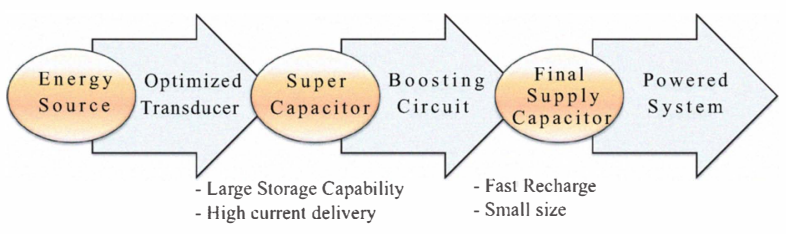

Fig. 1. Architecture of the wearable kinetic harvester.

circuitry to properly store the energy and to optimize their utilization [8].

Motion is definitely one of the most attractive energy sources for external wearable sensors $[9,10]$. The key challenge here is to effectively convert low-frequency kinetic energy into electric energy. Wristwatch makers have a long tradition in kinetic energy harvesting, but usually watches are mechanically powered. An interesting idea of a kinetic energy scavenger linked to an electrical power management and storage circuit, with reduced size and high wearability, is represented by the quartz wristwatch Seiko Kinetic [11,12]. Thanks to an oscillating weight coupled to a magnetic rotor through a gearbox, this watch can auto-recharge itself, storing the charge in a supercapacitor and transferring it in a smaller capacitor when needed, to make it immediately usable by the quartz circuit.

This auto-recharge system is very efficient and it has been successfully tested also in the biomedical field, charging a pacemaker battery by means of heart beats [13]. The circuitry behind this system consists of hundreds of logical gates integrated on an ASIC: any realization of this system on a different substrate, such as a printed circuit board using off-theshelf components, risks to drastically reduce its performance, decreasing the ratio between the power transferred from the supercapacitor to the smaller capacitor and the power required by the circuit to perform this transfer.

The aim of this work is to design a solution having the same characteristics and the same advantages of the one proposed by Seiko, but more suitable for physical realization on printed circuit boards thanks to a reduced logic complexity.

\section{HARVESTER DESIGN}

The goal of the proposed system is to store the charge collected by a wearable micro-engine in a supercapacitor and to transfer it into a smaller accumulator which can quickly reach power levels suitable for a sensor node, as shown in Fig. 1.

As mentioned in the introduction, if the harvested energy is stored into a supercapacitor which is directly connected to the load, the start-up time of the powered system is increased to several seconds or minutes. Supercapacitors are able to store a large amount of energy, but their time constant $\tau=R C$ is extremely high, due to their large capacitance value; for this reason, their voltage rises very slowly when they are charged by low currents. A useful voltage level to power the load is reached after a very long time and this is unacceptable in most applications. We propose a kinetic harvester which uses a

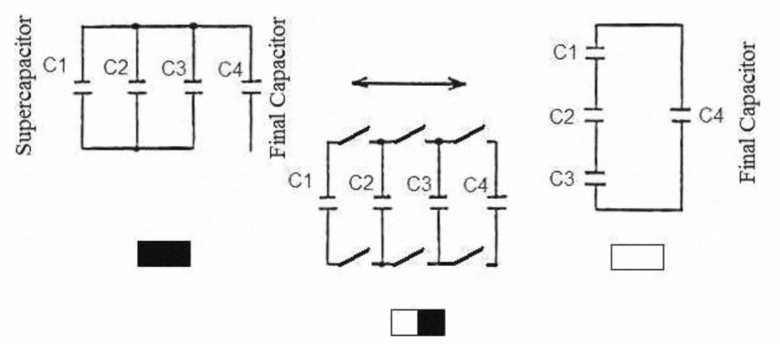

(a)

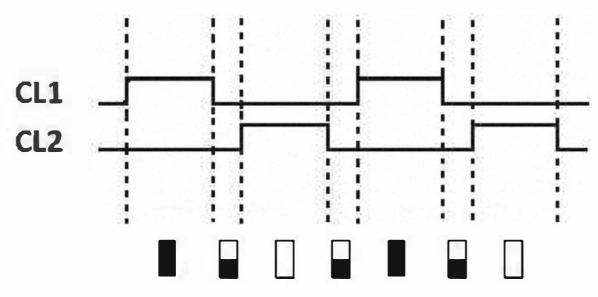

(b)

Fig. 2. Schematic functioning of a boosting circuit with multiplicative factor 3 .

supercapacitor as charge tank, exploiting its storage capability. The system transfers part of the collected charge into a smaller capacitor, where the voltage level increases faster, providing power supply directly to the load. The proposed approach reduces considerably the start-up time, if compared with harvesters equipped with a single supercapacitor as the only energy storage device.

\section{A. Boosting Circuit}

1) Description: The core of the energy harvester is the boosting circuit. Its task is to transfer the charge stored in the supercapacitor into a smaller one, named final capacitor. The literature offers a large amount of methods to realize charge pump circuits [14-17]: we have chosen a solution where the supercapacitor and the final capacitor are used together with one or more flying capacitors and the voltage multiplication is obtained by switching these capacitances in different configurations.

Fig. 2a shows an example of voltage multiplication with a multiplicative factor 3 : in the configuration on the left, flying capacitors $C_{2}$ and $C_{3}$ are in parallel with supercapacitor $C_{1}$ and they are charged to its voltage; in the configuration on the right, the series of $C_{1}, C_{2}$ and $C_{3}$ creates a voltage drop across the final capacitor $C_{4}$ equal to three times the voltage across the supercapacitor $C_{1}$. By switching between these two configurations, every switching cycle raises the voltage of the final capacitor $C_{4}$ of a $\delta V$ step, until a voltage triple of the one across the supercapacitor is reached (Fig. 3). The number of $\delta V$ steps and their amplitude depend on several parameters: value of the flying capacitors, value of the final capacitor, switching frequency.

The switching operation is driven by the clock signals CL1 and CL2, visible in Fig. 2b. These signals are almost in opposite phases, but there is a time interval when they are 


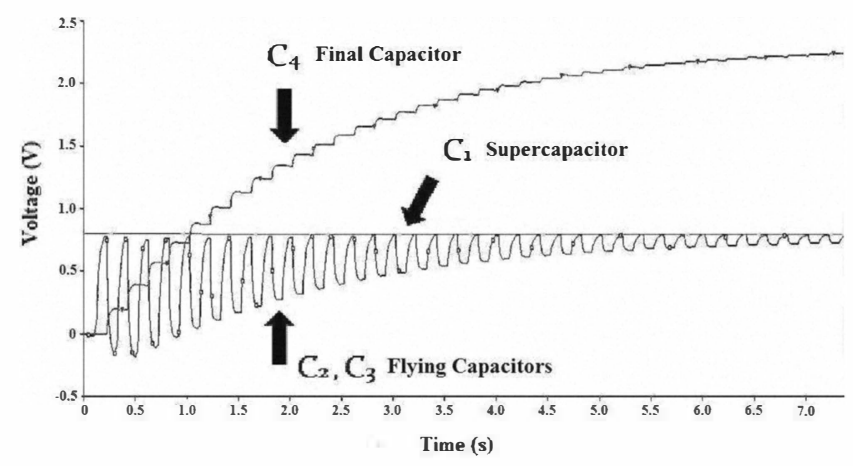

Fig. 3. Voltage across the different capacitors of the boosting circuit during a charge cycle.

both low: this precaution has been used to avoid unwanted intermediate configurations of the capacitances, due to possible skews of these signals, that could compromise the correct operation and the efficiency of the boosting circuit.

Theoretically, by means of the method described, using $n$ flying capacitors having the same value, all the following multiplicative factors can be obtained:

$$
\overline{M_{n}}=\left\{1 ; 1+\frac{k}{j} ; \overline{M_{n-1}}+1\right\}_{\substack{j=1 \ldots n \\ k=1 \ldots j-1}}
$$

where $\overline{M_{1}}=\{1 ; 2\}$.

To reduce system area, cost and complexity, we decided to use only two flying capacitors; the multiplicative factors obtainable are thus included in the vector $\overline{M_{2}}=\{1 ; 1.5 ; 2 ; 3\}$. Among these values, the biggest one has been selected to activate the system more quickly.

2) Sizing: The final capacitor $C_{4}$ must be able to store sufficient energy to complete the execution of tasks of prespecified duration by the sensor node, before $C_{4}$ is completely depleted and the node shuts down. Our reference sensor node is equipped with a MSP430 microcontroller by Texas Instruments: its supply voltage is in the range between $1.8 \mathrm{~V}$ and $3.6 \mathrm{~V}$ and the average power consumption of the whole node is around $400 \mu \mathrm{W}$, when executing a defined benchmark which includes $\mathrm{A} / \mathrm{D}$ conversions, Flash erasing, Flash writing and radio communication. If charged at $3.6 \mathrm{~V}$, a standard $470 \mu \mathrm{F}$ final capacitor can power the sensor node for a time of nearly $5.7 \mathrm{~s}$ while executing the benchmark, with no additional energy transferred from the supercapacitor into the final capacitor. This time is enough for the microcontroller to execute the benchmark and to shut down: thus, we have fixed at $470 \mu \mathrm{F}$ the value of the final capacitor.

The choice of the supercapacitor value $C_{1}$ involves a tradeoff: on one hand, it should be as large as possible to store more charge and to guarantee a longer autonomy to the circuit; on the other hand, a large value of capacitance increases the time period needed to reach a useful voltage level to start the voltage multiplication, thus increasing the start-up time of the sensor node. After several measurements, we have found

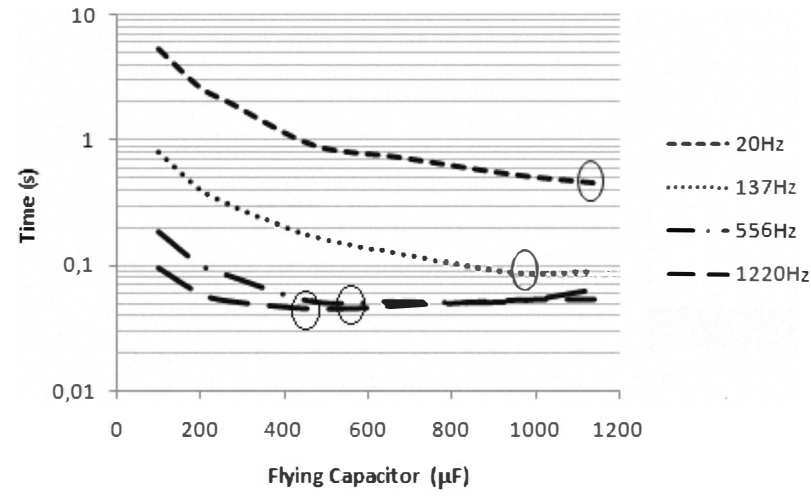

Fig. 4. Charging time as a function of the flying capacitors, for different switching frequencies. Final capacitor is $470 \mu \mathrm{F}$. Optimum values for any switching frequency have been pointed out by a circle.

that a commercially available $0.33 \mathrm{~F}$ value represents a good trade-off between autonomy and start-up time.

The flying capacitors $C_{2}$ and $C_{3}$ are chosen equal. Their optimum value is the one that minimizes the time needed by the final capacitor to reach a voltage level equal to 3 times the voltage across the supercapacitor. That value depends on the switching frequency and on the sizing of the final capacitor: a higher value of switching frequency, as well as a lower value of the final capacitor, implies the optimal value of the flying capacitors to be smaller.

Since the size of the final capacitor is fixed, we have experimentally obtained the optimum value of the flying capacitors for a wide range of switching frequencies. The charging time as a function of the value of the flying capacitors is shown in Fig. 4, where the optimum value for each frequency is highlighted by a circle.

Once the optimum size of the flying capacitors has been determined for a set of frequencies, we focus on the choice of the switching frequency. In Table I we report the average power transferred to the final capacitor in a boosting cycle (while the best value of flying capacitors is used) and the power drawn by the boosting circuit from the supply. It is easy to note that the average transferred power does not increase proportionally with the frequency as the power absorbed from the supply: while the value of the average transferred power seems to saturate when the switching frequency rises above $1 \mathrm{kHz}$, the power drawn from the supply increases in all the considered ranges.

TABLE I

POWER ANALYSIS DURING A BOOSTING CYCLE

\begin{tabular}{|c|c|c|}
\hline $\begin{array}{c}\text { Switching } \\
\text { Frequency }\end{array}$ & $\begin{array}{c}\text { Average Transferred } \\
\text { Power }\end{array}$ & $\begin{array}{c}\text { Power Absorbed } \\
\text { from Supply }\end{array}$ \\
\hline $20 \mathrm{~Hz}$ & $2.94 \mathrm{~mW}$ & $2.40 \mu \mathrm{W}$ \\
\hline $140 \mathrm{~Hz}$ & $15.4 \mathrm{~mW}$ & $3.60 \mu \mathrm{W}$ \\
\hline $550 \mathrm{~Hz}$ & $26.0 \mathrm{~mW}$ & $5.70 \mu \mathrm{W}$ \\
\hline $1220 \mathrm{~Hz}$ & $29.7 \mathrm{~mW}$ & $8.40 \mu \mathrm{W}$ \\
\hline $2270 \mathrm{~Hz}$ & $30.2 \mathrm{~mW}$ & $13.8 \mu \mathrm{W}$ \\
\hline
\end{tabular}




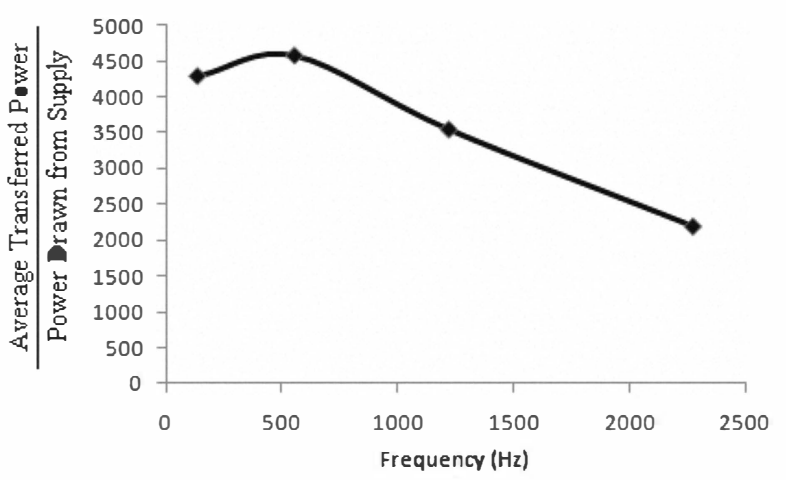

Fig. 5. Ratio between the average transferred power and the power drawn from the supply against the frequency.

The ratio between the average transferred power and the power drawn from the supply for any frequency in the range is plotted in Fig. 5. Notice that all the values in the observed range are sufficiently high, which assures the good operation of the boosting circuit; however, the function presents a maximum around $500 \mathrm{~Hz}$ and for higher frequencies the ratio quickly decreases. For this reason, we have fixed the switching frequency at $500 \mathrm{~Hz}$ and the value of the flying capacitors $C_{2}$ and $C_{3}$ at $470 \mu \mathrm{F}$.

\section{B. Harvester Operation}

The architecture of the energy scavenger is reported in Fig. 6. The part included in the shaded area represents the quick-start circuit and its operation will be described in the next paragraph: for a first analysis we will temporarily disregard it and the resistance $R_{1}$ will be considered as a short-circuit.

The comparator A, with internal threshold of $0.9 \mathrm{~V}$, monitors the voltage across the supercapacitor: its output, tagged "I can help you", provides an active high signal when the potential of the supercapacitor exceeds that threshold, meaning that it is ready to provide charge to the final capacitor by means of the boosting circuit.

The comparator $\mathrm{B}$, with internal threshold of $2.4 \mathrm{~V}$, monitors the voltage across the final capacitor: its output, tagged "Help me", provides an active high signal when the potential of the final capacitor is below that threshold, meaning that it needs to be helped by the supercapacitor, to be able to power the sensor node.

When both signals are high, the monostable circuit is unlocked and, driving high the signal "Boosting", it enables the boosting circuit for a period fixed by its internal parameters, regardless of the successive behavior of the signals "Help me" and "I can help you"; this time period is calibrated to allow the final capacitor to reach a voltage triple of the one of the supercapacitor.

To prevent the final capacitor to be charged over the maximum voltage of $3.6 \mathrm{~V}$ when the supercapacitor has a voltage

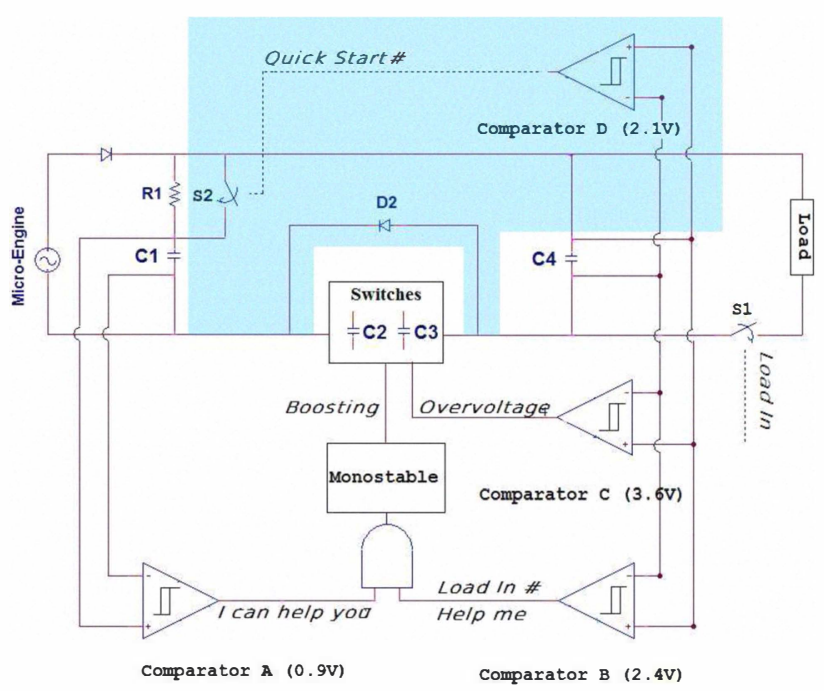

Fig. 6. Schematic of the whole energy scavenger.

higher than $1.2 \mathrm{~V}$, the comparator $\mathrm{C}$, with internal threshold of $3.6 \mathrm{~V}$, monitors the voltage across the final capacitor: its output is called "Overvoltage" and it gives an active high signal when the potential of the final capacitor exceeds that threshold, stopping the activity of the boosting circuit.

The output of comparator B is used also as signal "Load in \#": when it is low, acting on the switch $S_{1}$, it connects the sensor network to the supply provided by the final capacitor.

Finally, the Schottky diode $D_{1}$ rectifies the voltage generated by the micro-engine.

\section{Quick-Start Circuit}

The boosting circuit is powered by the same final capacitor used for the sensor node. For this reason, the final capacitor needs to be precharged in a defined setup time. The solution we used to overcome this issue, called quick-start circuit, involves some modifications in the schematic previously described: these changes are reported in the shaded area in Fig. 6.

Whenever the potential across the final capacitor is below $2.1 \mathrm{~V}$, the output signal "Quick-start \#" of comparator D is low, switch $S_{2}$ is opened and the boosting circuit is switched off: the equivalent circuit in this case is visible in Fig. 7.

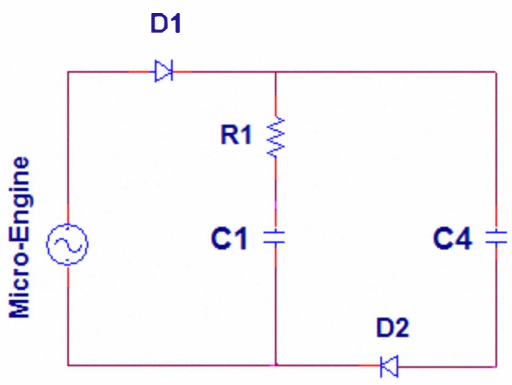

Fig. 7. Equivalent circuit when the "Quick-start \#" signal is low and the quick-start circuit is on. 


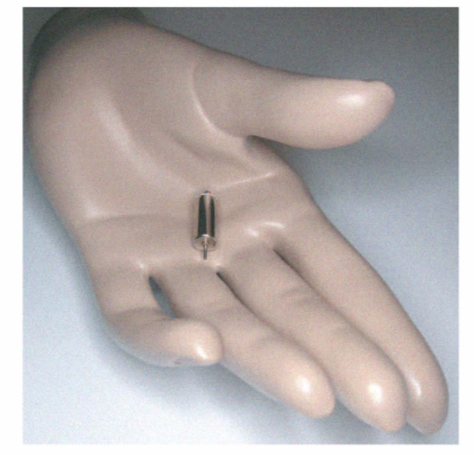

Fig. 8. Example of micro-engine suitable for wearable applications.

Disregarding the thresholds of the Schottky diodes, the voltage $V_{4}$ across the final capacitor $C_{4}$ is equal to $V_{1}+R_{1} I_{1}$, where $V_{1}$ is the voltage across the supercapacitor $C_{1}$ and $R_{1} I_{1}$ is the voltage drop caused by the current $I_{1}$ passing across the resistor $R_{1}$; the Schottky diode $D_{2}$ allows $C_{4}$ to charge itself, without permitting it to discharge if $I_{1}$ stops to flow on $R_{1}$.

When $V_{4}$ reaches $2.1 \mathrm{~V}$, the output signal "Quick-start \#" goes high closing the switch $S_{2}$ : resistor $R_{1}$ is thus bypassed by the switch and the charge generated by the micro-engine is completely used to charge the supercapacitor. The final capacitor is now steady at $2.1 \mathrm{~V}$, keeping high the signal "Help me" by means of the comparator $\mathrm{B}$, being ready to power the boosting circuit whenever the voltage across the supercapacitor rises over $0.9 \mathrm{~V}$, generating the signal "I can help you".

\section{Implementation}

The proposed system has been implemented using Commercial Off-the-Shelf (COTS) components on a printed circuit board. This makes the cost of the system affordable even with low volume production, without the need for complex and specialized ASICs. All the logical gates have Advanced Ultralow Power (AUP) features with very low dynamic and static power consumption (up to $30 \%$ less than standard CMOS gates) and an extremely compact package. Comparators belong to the S1000 family of Seiko, characterized by high precision detection voltage (accuracy of $\pm 1 \%$ ), ultra-low current consumption (few hundreds of $\mathrm{nA}$ ) and small package. The clock is generated by means of a comparator MAX919, having a minimum supply current (few hundreds of $\mathrm{nA}$ ), opportunely inserted in a feedback loop to create an ultra-low power oscillator.

The low power consumption of all the used components, together with the absence of a complex control logic to manage the functioning, increase the performance with a high ratio between the power transferred to the final capacitor and the power drawn from the supply (Fig. 5). Moreover, the compactness of their packages allows for assembly in miniaturized cases, typical of the wearable sensing nodes.

The powered sensor node is a miniaturized system designed to support wearability and unobtrusiveness with modular architecture where each functional block is included only if required
TABLE II

WAKE-UP TIMES

\begin{tabular}{|c|c|c|}
\hline $\begin{array}{c}\text { Stimulus } \\
\text { Amplitude }\end{array}$ & $\begin{array}{c}\text { Wake-up Time } \\
\text { Supercapacitor }\end{array}$ & $\begin{array}{c}\text { Wake-up Time } \\
\text { Energy Scavenger }\end{array}$ \\
\hline $3.6 \mathrm{~V}$ & $100 \mathrm{~s}$ & $20 \mathrm{~s}$ \\
\hline $3.2 \mathrm{~V}$ & $140 \mathrm{~s}$ & $20 \mathrm{~s}$ \\
\hline $2.8 \mathrm{~V}$ & $200 \mathrm{~s}$ & $30 \mathrm{~s}$ \\
\hline $2.5 \mathrm{~V}$ & $290 \mathrm{~s}$ & $85 \mathrm{~s}$ \\
\hline
\end{tabular}

by the application (e.g. some nodes would be equipped with specified sensors, other would only host radio for data routing and additional processing capability). The core of the sensor node is based on an ultra-low power RISC microcontroller (MSP430 from Texas Instruments) which provides an optimal tradeoff between power consumption and performance. Communication capabilities have been implemented using a low-power Zigbee radio. To meet low-power requirements the radio is used with an aggressive duty-cycle (below 1\%) which allows to achieve sub-mW power consumption.

\section{Measurements}

The experimental measurements reported in this section have the goal to compare the system proposed against a single supercapacitor acting both as power tank and as supply for the load. In these measurements, we have applied identical stimuli to the single supercapacitor and to the scavenger, observing the time needed by the two systems to reach a useful voltage to power the load; this time period has been named "wake-up time".

The load is a sensor node equipped with a microcontroller MSP430: its minimum supply voltage is $1.8 \mathrm{~V}$. The single supercapacitor is able to power it as soon as its voltage is stable over that threshold; instead, the scavenger needs to reach a value higher than $2.4 \mathrm{~V}$ across the final capacitor to be connected to the load and to power it. For this reason, the wake-up time of the single supercapacitor has been defined as the time needed by it to reach a stable voltage of $1.8 \mathrm{~V}$, while the wake-up time of the scavenger has been defined as the time needed by the final capacitor to reach $2.4 \mathrm{~V}$.

The stimuli, identical in both cases, emulate the output of a micro-motor moved by human activities. The micro-engine used to create those stimuli is manufactured by Precision Microdrives [18] and is shown in Fig. 8.

When the micro-engine is attached to the wrist, an average human motion frequency around $0.5 \mathrm{~Hz}$ can be easily achieved without an overload for the user. The amplitude of the generated stimulus depends on several factors: acceleration given to the shaft, amplifications by means of gearboxes, load impedance. The adopted micro-engine is able to return a variable output voltage between $1.6 \mathrm{~V}$ and $3.3 \mathrm{~V}$ when closed on load impedance between $45 \Omega$ and $45 \mathrm{k} \Omega$. The wake-up times obtained for different values of amplitude of the stimulus and a motion frequency of $0.5 \mathrm{~Hz}$ are shown in Table II. Notice that the scavenger is ready to power the load in a time period up to 7 times shorter than the one needed by the single supercapacitor. 


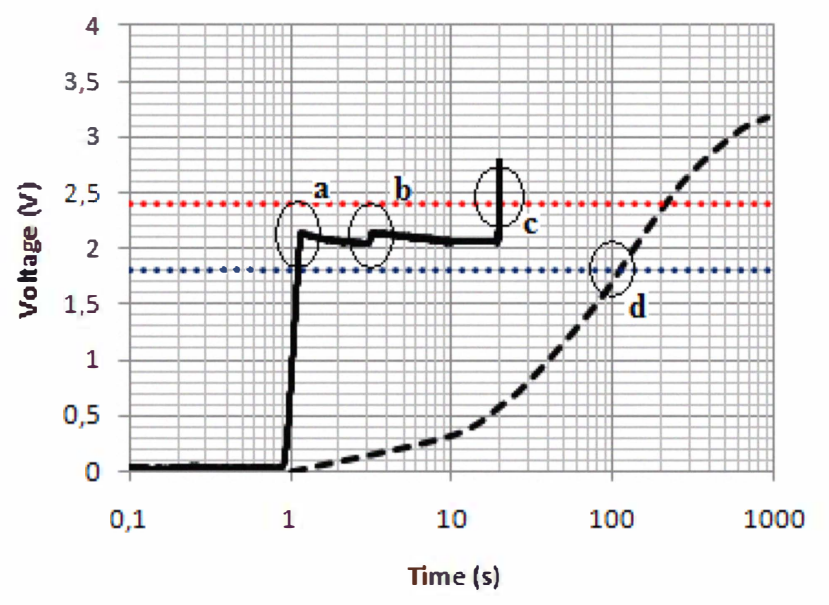

Fig. 9. Comparison between the charge obtained with the scavenger (continuous line) and with a single supercapacitor (dotted line). Wake-up time of the scavenger occurs at point $(c)$ while wake-up time of the single supercapacitor occurs at point $(d)$.

The comparison of the wake up time achieved using the classic approach and our solution is plotted also in Fig. 9. The stimulus is applied to both systems at the same time: the voltage trend across the final capacitor of the energy scavenger is reported as a continuous line, while the voltage trend of the single supercapacitor is reported as a dotted line. The voltage across the single supercapacitor slowly rises, whereas the voltage across the final capacitor of the scavenger immediately reaches the value $2.1 \mathrm{~V}$, thanks to the quickstart circuit. At point $(a)$ the quick-start circuit is switched off and the final capacitor slowly discharges itself to power the scavenger circuitry; at point $(b)$ the quick-start circuit is reactivated to permit the final capacitor to reach again a voltage of $2.1 \mathrm{~V}$; finally, at point $(c)$ the supercapacitor of the scavenger is charged enough to activate the boosting circuit and the voltage across the final capacitor quickly rises up to three times the voltage of the supercapacitor, crossing the value $2.4 \mathrm{~V}$ and powering the load. Point $(c)$ determines the wakeup time of the energy scavenger. The single supercapacitor instead needs more time to reach a useful voltage and it is able to power the load only at point $(d)$, that determines its wake-up time.

Another experimental comparison has been performed by increasing the duration of the incoming stimulus. Fig. 10 shows the results obtained using a sine wave with amplitude $3.2 \mathrm{~V}$ and frequency $0.5 \mathrm{~Hz}$ used to emulate the signal provided by the micro-engine of the scavenger. The shaded boxes illustrate the duration of the applied signal, while the lines indicate when the harvester (continuous lines) and the single supercapacitor (dotted lines) start and finish to power the sensor node. This plot shows that the scavenger can power the load exploiting even short stimuli, that are not enough for the single supercapacitor (e.g. cases with $120 \mathrm{~s}$ and $60 \mathrm{~s}$ ).

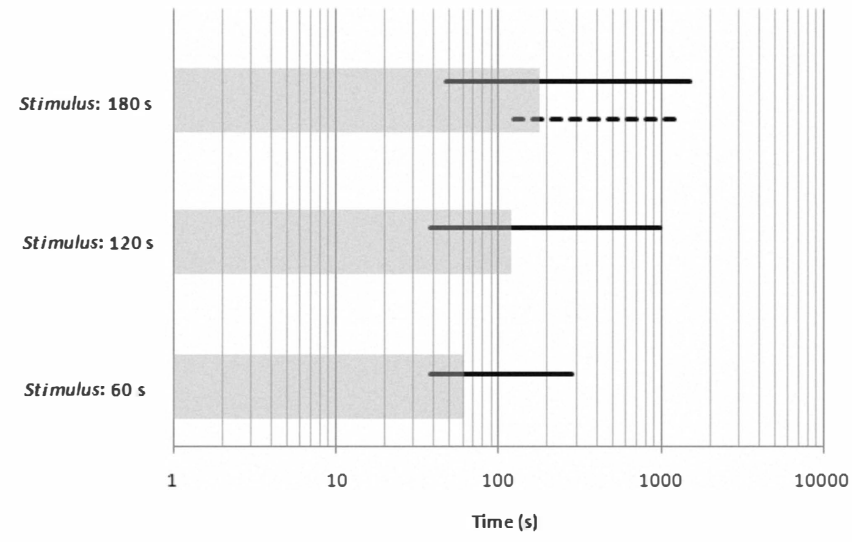

Fig. 10. Comparison between the behavior of the scavenger (continuous line) and of the single supercapacitor (dotted line) when excited with short stimuli (shaded areas).

\section{CONClusion}

We have proposed an energy scavenger which collects electric energy from a micro-engine used as a generator, allowing an immediate start-up of a sensor node. This scavenger does not need a dedicated control ASIC and it has been implemented with low-cost, off-the-shelf discrete components. The use of a boosting circuit to transfer in a final capacitor the charge collected into a supercapacitor allows to reach a useful voltage level to power a sensor node in a very short time, up to seven times faster than in the case where only a single supercapacitor is used as storage device.

The charge pump parameters, such as the value of the flying capacitors and the switching frequency, have been carefully optimized. Measurements done to validate the design, reported in Fig. 9 and Fig. 10, confirm the advantages obtained by the scavenger when compared with a single supercapacitor used as power supply. Indeed, the proposed system is able to power the node faster than the single supercapacitor; moreover, it is able to efficiently exploit short stimuli that are not sufficient to charge the supercapacitor above a useful threshold. Future work will investigate a further miniaturization of the device, with particular emphasis on wearability. Efficiency will be further improved using smart circuits which detect when the supercapacitor is completely charged and switch off the boosting system. This upgrade will reduce the power consumption, while preserving the gain obtained by the boosting circuit in terms of start-up time.

\section{ACKNOWLEDGMENTS}

The work presented in this paper has been founded by a grant of Telecom Italia. In addition, this research was partially supported by the ARTISTDesign Network of Excellence (IST2007-214373) of the EU 7th Framework Programme.

\section{REFERENCES}

[1] M. Kishi, H. Nemoto, T. Hamao, M. Yamamoto, S. Sudou, M. Mandai, and S. Yamamoto, "Micro-thermoelectric modules and their application 
to wristwatches as an energy source," in Proc. 18th Int. Conf. Thermoelectrics (ICT'99), Aug.-Sep. 29-2 1999, pp. 301-307.

[2] V. Leonov, T. Torfs, P. Fiorini, and C. Van Hoof, "Thermoelectric converters of human warmth for self-powered wireless sensor nodes," Sensors Journal, IEEE, vol. 7, no. 5, pp. 650-657, 2007.

[3] K. Goto, T. Nakagawa, O. Nakamura, and S. Kawata, "An implantable power supply with an optically rechargeable lithium battery," Biomedical Engineering, IEEE Transactions on, vol. 48, no. 7, pp. 830-833, 2001.

[4] M. Catrysse, B. Hermans, and R. Puers, "An inductive power system with integrated bi-directional data-transmission," Sensors and Actuators A: Physical, vol. 115, no. 2-3, pp. 221 - 229, 2004, the 17th European Conference on Solid-State Transducers.

[5] B. Lenaerts and R. Puers, "An inductive power link for a wireless endoscope," Biosensors and Bioelectronics, vol. 22, no. 7, pp. 1390 1395, 2007.

[6] S. Suzuki, T. Katane, H. Saotome, and O. Saito, "Electric powergenerating system using magnetic coupling for deeply implanted medical electronic devices," Magnetics, IEEE Transactions on, vol. 38, no. 5, pp. 3006-3008, 2002.

[7] S. Kerzenmacher, J. Ducre, R. Zengerle, and F. von Stetten, "Energy harvesting by implantable abiotically catalyzed glucose fuel cells," Journal of Power Sources, vol. 182, pp. 1-17, 2008.

[8] R. Vullers, R. van Schaijk, I. Doms, C. Van Hoof, and R. Mertens, "Micropower energy harvesting," Solid-State Electronics, vol. 53, no. 7 pp. $684-693,2009$.

[9] P. Mitcheson, E. Yeatman, G. Rao, A. Holmes, and T. Green, "Energy harvesting from human and machine motion for wireless electronic devices," Proceedings of the IEEE, vol. 96, no. 9, pp. 1457-1486, 2008.

[10] J. M. Donelan, Q. Li, V. Naing, J. A. Hoffer, D. J. Weber, and A. D. Kuo, "Biomechanical energy harvesting: Generating electricity during walking with minimal user effort," Science, vol. 319, no. 5864, pp. 807810, 2008

[11] European Patent Office Patent $0326313,1989$.

[12] M. Hayakawa, "A study of the new energy system for quartz watches (ii) - the effective circuit for the system," in Congrs europen de Chronomtrie, 1988.

[13] H. Goto, T. Sugiura, Y. Harada, and T. Kazui, "Feasibility of using the automatic generating system for quartz watches as a leadless pacemaker power source," Medical and Biological Engineering and Computing, vol. 37, no. 1, pp. 377-380, Jan. 1999.

[14] J.Dickson, "On-chip high-voltage genertion in nmos integrated circuits using an improved voltage multiplier technique," IEEE Journal of SolidState Circuits, vol. 11, no. 6, pp. 374-378, June 1976.

[15] J. Wu and K. Chang, "Mos charge pumps for low-voltage operation," IEEE Journal of Solid-State Circuits, vol. 33, no. 4, pp. 592-597, April 1998.

[16] J. Silva-Martinez, "A switched capacitor double voltage generator," in Circuits and Systems, 1994., Proceedings of the 37th Midwest Symposium on, vol. 1, 1994, pp. 177-180 vol.1.

[17] P. Favrat, P. Deval, and M. Declercq, "A high-efficiency cmos voltage doubler," IEEE Journal of Solid-State Circuits, vol. 33, no. 3, pp. 410416, March 1998.

[18] 7mm micro dc motor. http://www.precisionmicrodrives.com/. 